\title{
Paroxysmal atrial fibrillation in cryptogenic stroke
}

This article was published in the following Dove Press journal:

Therapeutics and Clinical Risk Management

27 January 201 I

Number of times this article has been viewed

\author{
Neha S Dangayach' \\ Kevin Kane ${ }^{2}$ \\ Majaz Moonis ${ }^{3}$ \\ 'Saint Vincent Hospital, ${ }^{2}$ University \\ of Massachusetts Medical School, \\ ${ }^{3}$ University of Massachusetts \\ Memorial Health Center, Worcester, \\ MA, USA
}

Introduction: Paroxysmal atrial fibrillation (PAF) is perhaps the most underdiagnosed mechanism of apparent cryptogenic stroke (CS). Various studies have shown that increasing the duration of monitoring can increase the diagnosis of PAF in CS.

Methods: We compared demographic and risk factors for ischemic stroke across different TOAST (Trial of ORG 10172 in Acute Stroke Treatment) stroke subtypes to look for clinical differences between other subtypes and CS and subsequently performed periodic Holter monitoring and imaging studies in apparent CS patients.

Results: Of the 298 patients with ischemic stroke, 17\% had CS. Periodic holter monitoring enabled diagnosis of PAF in $29 \%$ of patients. Five of 51 patients with CS had recurrent ischemic stroke and all 5 were demonstrated as PAF on repeated Holter monitoring.

Conclusions: Long-term periodic rhythm monitoring in patients with apparent CS showed PAF in a significant percentage of CS patients, which altered subsequent treatment.

Keywords: cryptogenic stroke, atrial fibrillation, stroke of undetermined etiology

\section{Introduction}

Understanding the etiological basis of acute ischemic stroke is important in optimizing treatment to reduce the chances of recurrent ischemic stroke (RIS). ${ }^{1}$ Warfarin is the accepted treatment for cardioembolic stroke, ${ }^{2}$ while antiplatelet drugs with or without carotid endartectomy or carotid artery stenting are the appropriate treatment for large artery atherosclerosis (LAA). ${ }^{3}$

Even with the most comprehensive in-patient evaluation, $20 \%$ to $30 \%$ of ischemic stroke has undefined etiologies: cryptogenic stroke (CS). ${ }^{4-8}$ These patients are often randomly assigned to treatment options depending upon the perceived etiology of the stroke by their physician. The American Heart Association/American Stroke Association (AHA/ASA) guidelines $^{9}$ help in identifying structural causes for stroke such as atherothrombosis or known cardiac causes (eg, known atrial fibrillation, low ejection fraction).

However, one of the most neglected aspects of stroke evaluation is intermittent rhythm disorders such as paroxysmal atrial fibrillation (PAF), which may not be evident within the suggested 24-hour telemetry period and is as important as chronic atrial fibrillation (CAF) as a risk factor for stroke. Therefore, we prospectively investigated the prevalence of PAF in apparent CS.

\section{Methods}

Patients with a first-ever ischemic stroke admitted to the University of Massachusetts Memorial Health Center were worked up as per the standard ASA/AHA acute care 
guidelines $^{9}$ for a period of 7 years from 2002 to 2009 and were classified into subtypes according to TOAST (Trial of ORG 10172 in Acute Stroke Treatment) criteria and definitions. ${ }^{1}$ Transient ischemic attacks (TIA), while not a part of the TOAST classification, were nevertheless included since they constitute a high risk group for subsequent stroke and may have PAF or CAF as an underlying mechanism. The study remains an ongoing project and data collected between 2002 and 2009 were analyzed. Patients seen in the stroke prevention clinic with a diagnosis of CS were included in the study for periodic follow-ups. Patients with hemorrhagic stroke were excluded. The entire cohort of patients was transitioned from the inpatient service to follow-up in the stroke prevention clinic. All patients had a transthorasic echocardiogram (TTE) and those with normal TTE and no other obvious risk factors underwent a TEE to assess for left atrium, auricle, and aortic arch disease. Patients were classified as CS only after a full extensive work-up including cardiac studies, hypercoagulable studies, continued inpatient telemetry where indicated and computed tomography (CT) and CT angiogram followed by magnetic resonance imaging (MRI) of the brain where noncontrast CT was normal.

\section{Protocol}

At each 6-monthly visit, a comprehensive evaluation and medical reconciliation were performed. The evaluation consisted of fasting lipid panel, glucose, C-reactive protein, Doppler studies of the neck, and 48-hour Holter monitoring.

PAF was defined as episodes of AF that lasted for less than 1 week and resolved spontaneously. ${ }^{10}$ To detect PAF, patients underwent periodic 48-hour Holter monitoring every 6 months. Stroke-related risk factors (hypertension, diabetes mellitus, hyperlipidemia, past history of myocardial infarction [MI], coronary artery disease [CAD]), demographics, and medication use were compared across different subtypes to assess for possible clinical differences between patients with CS and other stroke subtypes.

A subgroup analysis of all patients determined to have cardioembolic stroke (including newly diagnosed PAF and CAF) was performed to look for possible clinical similarities and differences between patients with CS with later defined PAF and those presenting as obvious cardioembolic stroke at onset. We also compared the subgroup of patients with CS and PAF and the subgroup of patients with CS without PAF to identify any differences that might again help to predict which patients with CS have an underlying cardiac arrhythmia, namely PAF.

\section{Statistical methods}

One way analysis of variance (age) and Chi-square tests (risk factors, medications, gender) were used to compare the variables in the different groups, and a $P$-value $<0.05$ was regarded as significant.

\section{Results}

Two hundred ninety-eight patients with ischemic stroke were evaluated. The mean age of the subjects was $63.16 \pm 15.85$ years, and $56.7 \%$ of the patients with ischemic stroke were males. Subtype classification showed 54 (17\%) cardioembolic stroke, 74 (25\%) LAA, 87 (29\%) small artery disease, $51(17 \%)$ CS, and 32 (11\%) TIA. A comparative analysis of demographics and risk factors including hypertension, hyperlipidemia, CAD, diabetes mellitus, past history of $\mathrm{MI}$, and past history of TIA, yielded significant difference in age between the 5 groups $(P=0.043)$ (Table 1). A post-hoc Tukey test showed that patients with SAD were older than patients with CS $(P=0.076)$, but this difference did not reach significance. There were no other significant differences between the groups. Two patients with initial CS developed early subsequent PAF and therefore for

Table I Demographic and risk factors compared across the 5 subgroups of ischemic stroke and transient ischemic attack

\begin{tabular}{|c|c|c|c|c|c|c|}
\hline & LAA & CE & SAD & TIA & CS & $P$-value \\
\hline Age & $60.59 \pm 14.94$ & $65.66 \pm 17.33$ & $66.03 \pm 14.73$ & $64.89 \pm 16.46$ & $58.22 \pm 15.94$ & 0.043 \\
\hline \multirow[t]{4}{*}{ Gender } & 29 (39.2\%) & $22(40.7 \%)$ & 40 (46.0\%) & $9(28.1 \%)$ & 29 (56.9\%) & 0.102 \\
\hline & Female & Female & Female & Female & Female & \\
\hline & $45(60.8 \%)$ & 32 (59.3\%) & 47 (54.0\%) & 23 (7I.9\%) & $22(43.1 \%)$ & \\
\hline & Male & Male & Male & Male & Male & \\
\hline HTN & $40(54.1 \%)$ & 29 (53.7\%) & 49 (56.3\%) & I8 (56.3\%) & I8 (35.3\%) & 0.149 \\
\hline Hyper-lipidemia & 42 (56.8\%) & 27 (50.0\%) & 42 (48.3\%) & $16(50.0 \%)$ & 24 (47.1\%) & 0.813 \\
\hline Previous MI & 7 (9.5\%) & $4(7.4 \%)$ & $4(4.6 \%)$ & $2(6.3 \%)$ & $3(6.0 \%)$ & 0.805 \\
\hline A-Fib & $2(3.8 \%)$ & 14 (35.0\%) & $5(8.6 \%)$ & $4(14.8 \%)$ & $2(7.4 \%)$ & $<0.001$ \\
\hline Diabetes & 14 (I8.9\%) & II (20.4\%) & 15 (I7.2\%) & $5(15.6 \%)$ & $8(16.0 \%)$ & 0.965 \\
\hline CAD & 9 (12.2\%) & 10 (18.5\%) & $9(10.3 \%)$ & 7 (21.9\%) & 7 (I5.7\%) & 0.446 \\
\hline
\end{tabular}

Abbreviations: $\mathrm{AF}$, atrial fibrillation; $\mathrm{CAD}$, coronary artery disease; $\mathrm{CE}$, cardioembolic stroke; CS, cryptogenic stroke; HTN, hypertension; LAA, large artery atherosclerotic stroke; $\mathrm{MI}$, myocardial infarction; $\mathrm{SAD}$, small artery disease; TIA, transient ischemic attack. 
Table 2 Medication use compared across the 5 subgroups of ischemic stroke and transient ischemic attack

\begin{tabular}{lllllll}
\hline & LAA & CE & SAD & TIA & CS & P-value \\
\hline Antiplatelets & $49(66.2 \%)$ & $22(40.7 \%)$ & $6 I(70.1 \%)$ & $23(71.9 \%)$ & $27(52.9 \%)$ & 0.003 \\
ASA & $25(33.8 \%)$ & $19(35.2 \%)$ & $26(29.9 \%)$ & $13(40.6 \%)$ & $17(33.3 \%)$ & 0.860 \\
$\begin{array}{l}\text { ASA/extended-release } \\
\text { dipyridamole }\end{array}$ & $21(28.4 \%)$ & $3(5.6 \%)$ & $26(29.9 \%)$ & $8(25.0 \%)$ & $9(17.6 \%)$ & 0.008 \\
Clopidogrel & $12(16.2 \%)$ & $4(7.4 \%)$ & $9(10.3 \%)$ & $5(15.6 \%)$ & $7(13.7 \%)$ & 0.565 \\
Warfarin & $10(13.5 \%)$ & $24(44.4 \%)$ & $4(4.6 \%)$ & $7(21.9 \%)$ & $7(13.7 \%)$ & $<0.001$ \\
Statins & $43(58.1 \%)$ & $31(57.4 \%)$ & $51(58.6 \%)$ & $14(43.8 \%)$ & $21(41.2 \%)$ & 0.184 \\
ACE-Inhibitors & $24(32.4 \%)$ & $21(38.9 \%)$ & $31(35.6 \%)$ & $9(28.1 \%)$ & $13(25.5 \%)$ & 0.596 \\
Other Anti-HTN & $21(28.4 \%)$ & $27(50.0 \%)$ & $32(36.8 \%)$ & $12(37.5 \%)$ & $16(31.4 \%)$ & 0.138 \\
\hline
\end{tabular}

Abbreviations: ACE, angiotensin-converting enzyme; ASA, aspirin; CE, cardioembolic stroke; CS, cryptogenic stroke; HTN, hypertensives; LAA, large artery atherosclerotic stroke; SAD, small artery disease; TIA, transient ischemic attack.

demographic purposes were kept in the CS category because at the time of stroke they did not have AF. Comparison between known cardioembolic stroke and CS did not yield any features that could predict or distinguish between the 2 groups (Table 3 ).

The mean age of patients with CS in our study was $58.22 \pm 15.94$ years and was not significantly lower than that for other stroke subtypes including CE strokes. We compared the medications that the patients were on at the time of their initial visit to the clinic. As was expected, the use of medications differed among patients (Table 2).

During the follow-up of patients with CS, based on 48-hour Holter monitoring, 29\% patients were diagnosed with PAF within a period of 1 year from initial evaluation. Patients with brief $<30$-second nonrecurring PAF were not included in the analysis. There were no significant demographic differences among groups.

RIS was seen in 5 patients ( $10 \%$ of all CS patients). All were associated with newly diagnosed PAF. No patient with RIS was on warfarin. MRI in 4 of these patients also showed bihemispheric subcortical infarcts vessel consistent with an embolic etiology for stroke.

Table 3 Comparison of demographics, risk factors, and stroke recurrence in patients with cryptogenic stroke with and without paroxysmal atrial fibrillation

\begin{tabular}{llll}
\hline & PAF present & PAF absent & P-value \\
\hline $\mathrm{N}$ & $\mathrm{I}(29.4 \%)$ & $36(70.6 \%)$ & \\
Age & $61.53 \pm 16.28$ & $56.74 \pm 15.85$ & 0.375 \\
Gender & $8(53.3 \%)$ Male & $14(38.9 \%)$ Male & $0.37 \mathrm{I}$ \\
& $7(46.7 \%)$ Female & $22(61.1 \%)$ Female & \\
HTN & $6(40.0 \%)$ & $12(33.3 \%)$ & $0.75 \mathrm{I}$ \\
Hyperlipidemia & $8(53.3 \%)$ & $16(44.4 \%)$ & 0.759 \\
Previous MI & I (6.7\%) & $2(5.6 \%)$ & 1.000 \\
Diabetes & $4(26.7 \%)$ & $4(11.1 \%)$ & 0.213 \\
RIS & $5(33.3 \%)$ & 0 & 0.001 \\
CAD & $4(26.7 \%)$ & $4(I 1.1 \%)$ & 0.213 \\
\hline
\end{tabular}

Note: *No comparison was made for the number.

Abbreviations: CAD, coronary artery disease; HTN, hypertension; MI, myocardial infarction; PAF, paroxysmal atrial fibrillation, RIS, recurrent ischemic stroke.

\section{Discussion}

$\mathrm{AF}$ and atrial flutter account for about $10 \%$ of all strokes and $50 \%$ of cardioembolic strokes. ${ }^{11,12}$ Patients with PAF have many episodes of asymptomatic AF. ${ }^{13,14}$ Both CAF and PAF are comparable for their risk of stroke. ${ }^{14,15}$ In patients with CS periodic rhythm monitoring may identify new PAF in approximately 1 in 20 patients. $^{16}$

The incidence of CS in our study was $17 \%$. Other population-based studies have shown the incidence of CS to be $30 \%$ to $40 \% \%^{5-8,17}$ which may be attributed to different sampling methods.

Based on the treating physician's perception of the stroke subtype, patients with CS are often arbitrarily treated with warfarin or antiplatelet agents, as in our study (warfarin 14\% and antiplatelet agents 53\%).

We did not find any demographic differences between different ischemic stroke subtypes in spite of some studies suggesting that demographic data and risk factors may be significantly different across subtypes. ${ }^{17,18}$ Patients who had $\mathrm{CS}$ and were diagnosed with PAF had a higher incidence of diabetes, CAD, and past history of MI than CS patients without a diagnosis of PAF. Tayal et al also found that the incidence of diabetes was higher in patients of CS/TIA diagnosed with AF on mobile cardiac outpatient telemetry (MCOT). ${ }^{19}$ However these differences, while important, are not predictive of stroke subtype and are not helpful in individual treatment decisions.

$\mathrm{AF} /$ atrial flutter is a strong risk factor for stroke and is arguably the most important finding on cardiac work-up in patients with stroke. Treatment with warfarin and other oral anticoagulants provides a $44 \%$ greater relative risk reduction in recurrent stroke compared with antiplatelet therapy. ${ }^{16}$ In spite of the importance of diagnosing PAF they are perhaps the most under-studied mechanisms causing CS and no definitive guidelines are yet available for the follow-up and diagnoses of these disorders in patients with apparent CS. With periodic 6-monthly Holter monitoring, we found PAF 
in $29 \%$ of our CS cases within a year, which changed the treatment in these patients from antiplatelet drugs to warfarin. Interestingly, all 5 RIS that occurred during the follow-up period were all in patients with $\mathrm{CS}$, and delayed detection of PAF.

Different short-term follow-up strategies for diagnosing underlying PAF, such as automatic cardiac event recorders, ${ }^{20}$ 7-day external loop recorders, ${ }^{21}$ and 21-day MCOT, ${ }^{19}$ have been studied. Barthelemy et al monitored CS and TIA patients continuously using automatic cardiac event recorders for a mean period of $70.1 \pm 30.9$ hours and found PAF in 4 out of $28(14.3 \%)$ patients with CS. Approximately 33\% of AF episodes were missed in spite of the currently recommended thorough ECG and Holter monitoring. ${ }^{20}$ Jabaudon et al also found that external loop recorders for 7 days after acute stroke or TIA increased the detection of PAF and CAF by $5.7 \%$ $(5 / 88)$ patients. ${ }^{21}$ Tayal et al found that 21 -day MCOT enabled the diagnosis of $23 \%$ patients of CS/TIA with asymptomatic AF. ${ }^{19}$ However, unlike our study, they did not follow patients for a prolonged period after the acute ischemic stroke/TIA and may have missed some patient with potential cardioembolic stroke. Because the natural history of PAF is unknown, it is difficult to state what the optimal duration of monitoring should be in patients with CS/TIA to diagnose PAF. ${ }^{22}$

In our study, RIS was seen exclusively in the CS with subsequent PAF. This underscores the importance of proper identification of stroke subtypes for initiating appropriate strategies for secondary prevention such oral anticoagulants in ischemic stroke patients with PAF.

While there may be no differentiating clinical features to distinguish CS patients who have PAF, some ECG findings seem to be more informative. Kolb et al, in a small study of 33 patients where 297 episodes of PAF were detected using 12-lead Holter monitoring, found that most of these episodes were triggered by monomorphic left atrial premature complexes..$^{23,24,25}$ Similarly in some studies, patients with left atrial enlargement, spontaneous echo contrast were found to have a higher likelihood of PAF and cardioembolic events. ${ }^{26,27}$ Some studies have identified patients with PAF poststroke that was short lived and associated with an intracerebral hematoma. The authors argue that these short-lived episodes of AF may be triggered by the stroke itself. However if there is no other explanation for the etiology of stroke and it happens to be ischemic, ${ }^{28}$ PAF would still rank highly on the list as the underlying cause.

In conclusion, it seems that the present guidelines for rhythm monitoring for ischemic stroke are insufficient for detecting PAF, as shown by several studies as well as our own study. Increasing the duration of initial rhythm monitoring along with periodic rhythm monitoring (either with Holter or poststroke 30-day event monitoring) may increase the yield of PAF in CS. Based on our and other studies, to optimize detection of PAF, periodic 3- to 6-month monitoring seems reasonable, especially if there is history of intermittent palpitations, radiological evidence of bilateral infarcts, or ECG evidence of monomorphic premature atrial contractions without significant cardiac or atherosclerotic disease. A recent ongoing randomized prospective trial using standard arrhythmia monitoring versus an implantable device with a follow-up of 6 to 12 months may further enhance our knowledge of the prevalence of PAF in CS. ${ }^{29}$

\section{Disclosure}

This study was approved by the Institutional Review Board.

The authors have no conflicts of interest to disclose.

The study was prospectively designed and data were collected from the stroke prevention clinic. The study was not supported by any funding agency or pharmaceutical company.

\section{References}

1. Adams HP Jr, Bendixen BH, Kappelle LJ, et al. Classification of subtype of acute ischemic stroke. Definitions for use in a multicenter clinical trial. TOAST. Trial of Org 10172 in Acute Stroke Treatment. Stroke. 1993;24(1):35-41.

2. Rockson SG, Albers GW. Comparing the guidelines: anticoagulation therapy to optimize stroke prevention in patients with atrial fibrillation. J Am Coll Cardiol. 2004;43(6):929-935.

3. Ricotta JJ, Malgor RD. A review of the trials comparing carotid endarterectomy and carotid angioplasty and stenting. Perspect Vasc Surg Endovasc Ther. 2008;20(3):309-310.

4. Grau AJ, Weimar C, Buggle F, et al. Risk factors, outcome, and treatment in subtypes of ischemic stroke: the German stroke data bank. Stroke. 2001;32(11):2559-2566

5. Kolominsky-Rabas PL, Weber M, Gefeller O, Neundoerfer B, Heuschmann PU. Epidemiology of ischemic stroke subtypes according to TOAST criteria: incidence, recurrence, and long-term survival in ischemic stroke subtypes: a population-based study. Stroke. 2001;32(12):2735-2740.

6. Petty GW, Brown RD Jr, Whisnant JP, Sicks JD, O'Fallon WM, Wiebers DO. Ischemic stroke subtypes: a population-based study of incidence and risk factors. Stroke. 1999;30(12):2513-2516.

7. Sacco RL, Ellenberg JH, Mohr JP, et al. Infarcts of undetermined cause: the NINCDS Stroke Data Bank. Ann Neurol. 1989;25(4):382-390.

8. Schulz UG, Rothwell PM. Differences in vascular risk factors between etiological subtypes of ischemic stroke: importance of population-based studies. Stroke. 2003;34(8):2050-2059.

9. Adams HP Jr, del Zoppo G, Alberts MJ. Guidelines for the early management of adults with ischemic stroke: a guideline from the American Heart Association/American Stroke Association Stroke Council, Clinical Cardiology Council, Cardiovascular Radiology and Intervention Council, and the Atherosclerotic Peripheral Vascular Disease and Quality of Care Outcomes in Research Interdisciplinary Working Groups: The American Academy of Neurology affirms the value of this guideline as an educational tool for neurologists. Stroke. 2007;38(5):1655-16711. 
10. Lévy S, Novella P, Richard P, Pagenella F. Paroxysmal atrial fibrillation: a need for classification. J Cardiovasc Electrophysiol. 1995; 6(1):69-74.

11. Wolf PA, Abott RD, Kannel WB. Atrial fibrillation as an independent risk factor for stroke. The Framingham Study. Stroke. 1991;22(8): 983-988.

12. Wolf PA, Dawber TR, Thomas HE Jr, Kannel WB. Epidemiological assessment of chronic atrial fibrillation and risk of stroke: The Framingham study. Neurology. 1978;28(10):973-977.

13. Al-Khatib SM, Wilkinson WE, Sanders LL, McArthy EA0. Observations on transition from intermittent to permanent atrial fibrillation. Am Heart J. 2000;140(1):142-145.

14. Alpert JS, Petersen P, Godtfredsen J. Atrial fibrillation: natural history, complications and management. Annu Rev Med. 1988;39:41-52.

15. Marini C, de Santis F, Sacco S, et al. Contribution of atrial fibrillation to incidence and outcome of stroke: results from a population-based study. Stroke. 2005;36(6):1115-1119.

16. Liao J, Khalid Z, Scallan C, Morillo C, O'Donnell M. Noninvasive cardiac monitoring for detecting paroxysmal atrial fibrillation or flutter after acute ischemic stroke. A systematic review. Stroke. 2007; 38(11):2935-2940.

17. Lee BI, Nam HS, Heo JH, Kim DI, Yonsei Stroke Team. Yonsei Stroke Registry. Analysis of 1,000 patients with acute cerebral infarctions. Cerebrovasc Dis. 2001;12(3):145-151.

18. Schneider AT, Kissela B, Woo D, et al. Ischemic stroke subtypes: a population-based study of incidence rates among blacks and whites. Stroke. 2004;35(7):1552-1556.

19. Tayal AH, Tian M, Kelly KM, et al. Atrial fibrillation detected by mobile cardiac outpatient telemetry in cryptogenic TIA or stroke. Neurology. 2008;71(21):1696-1701.

20. Barthelemy JC, Feason-Gerard S, Garnier P, et al. Automatic cardiac event recorders reveal paroxysmal atrial fibrillation after unexplained strokes or transient ischemic attacks. Ann Noninvasive Electrocardiol. 2003;8(3):194-199.
21. Jabaudon D, Sztajzel J, Sievert K, Landis T, Sztajzel R. Usefulness of ambulatory 7-day ECG monitoring for the detection of atrial fibrillation and flutter after acute stroke and transient ischemic attack. Stroke. 2004;35(7):1647-1651.

22. Israel CW, Gronefeld G, Ehrlich JR, Li YG, Hohnloser SH. Long-term risk of recurrent atrial fibrillation as documented by an implantable monitoring device: implications for optimal patient care. J Am Coll Cardiol. 2004;43(1):47-52.

23. Kolb C, Nürnberger S, Ndrepepa G, Zrenner B, Schömig A, Schmitt C. Modes of initiation of paroxysmal atrial fibrillation from analysis of spontaneously occurring episodes using a 12-lead Holter monitoring system. Am J Cardiol. 2001;88(8):853-857.

24. Suttorp MJ, Kingma JH, Koomen EM, van't Hof A, Tijssen JG, Lie KI. Recurrence of paroxysmal atrial fibrillation or flutter after successful cardioversion in patients with normal left ventricular function. Am J Cardiol. 1993;71(8):710-713.

25. Rothman SA, Laughlin JC, Seltzer J. The diagnosis of cardiac arrhythmias: A prospective multi-center randomized study comparing Mobile cardiac outpatient telemetry versus standard loop event monitors. J Cardiovasc Electrophysiol. 2007;18(3):241-147.

26. Albers GW, Comess KA, DeRook FA, et al. Transesophageal echocardiographic findings in stroke subtypes. Stroke. 1994;25(1) 23-28.

27. Cerrato P, Imperiale D, Priano L, et al. Transesophageal echocardiography in patients without arterial and major cardiac sources of embolism: difference between stroke subtypes. Cerebrovasc Dis. 2002;13(3):174-183.

28. Vingerhoets F, Bogousslavsky J, Regli F, van Melle G. Atrial fibrillation after acute stroke. Stroke. 1993;24(1):26-30.

29. Sinha AM, Diener HC, Morillo CA, et al. Cryptogenic Stroke and underlying Atrial Fibrillation (CRYSTAL AF): design and rationale. Am Heart J. 2010;160(1):36-41.e1.
Therapeutics and Clinical Risk Management

\section{Publish your work in this journal}

Therapeutics and Clinical Risk Management is an international, peerreviewed journal of clinical therapeutics and risk management, focusing on concise rapid reporting of clinical studies in all therapeutic areas, outcomes, safety, and programs for the effective, safe, and sustained use of medicines. This journal is indexed on PubMed Central, CAS,

\section{Dovepress}

EMBase, Scopus and the Elsevier Bibliographic databases. The manuscript management system is completely online and includes a very quick and fair peer-review system, which is all easy to use. Visit http://www.dovepress.com/testimonials.php to read real quotes from published authors. 